\title{
Enhancing Rehabilitative Therapies with Vagus Nerve Stimulation
}

\author{
Seth A. Hays ${ }^{1,2,3}$ (D)
}

Published online: 15 December 2015

(C) The American Society for Experimental NeuroTherapeutics, Inc. 2015

\begin{abstract}
Pathological neural activity could be treated by directing specific plasticity to renormalize circuits and restore function. Rehabilitative therapies aim to promote adaptive circuit changes after neurological disease or injury, but insufficient or maladaptive plasticity often prevents a full recovery. The development of adjunctive strategies that broadly support plasticity to facilitate the benefits of rehabilitative interventions has the potential to improve treatment of a wide range of neurological disorders. Recently, stimulation of the vagus nerve in conjunction with rehabilitation has emerged as one such potential targeted plasticity therapy. Vagus nerve stimulation (VNS) drives activation of neuromodulatory nuclei that are associated with plasticity, including the cholinergic basal forebrain and the noradrenergic locus coeruleus. Repeatedly pairing brief bursts of VNS sensory or motor events drives robust, event-specific plasticity in neural circuits. Animal models of chronic tinnitus, ischemic stroke, intracerebral hemorrhage, traumatic brain injury, and post-traumatic stress disorder benefit from delivery of VNS paired with successful trials during rehabilitative training. Moreover, mounting evidence from pilot clinical trials provides an initial indication that VNS-based targeted plasticity therapies may be effective in patients with neurological diseases and injuries. Here, I provide a discussion of the current uses and potential future
\end{abstract}

Seth A. Hays

sxh129730@utdallas.edu

1 Texas Biomedical Device Center, Richardson, TX, USA

2 Erik Jonsson School of Engineering and Computer Science, The University of Texas at Dallas, Richardson, TX, USA

3 School of Behavioral Brain Sciences, The University of Texas at Dallas, Richardson, TX, USA applications of VNS-based targeted plasticity therapies in animal models and patients, and outline challenges for clinical implementation.

Keywords Neuroplasticity · Vagus nerve stimulation · Vagal nerve stimulation $\cdot$ Rehabilitation $\cdot$ Stroke $\cdot$ Tinnitus

\section{Introduction}

Pathological activity in neural circuits resulting from disease or injury can impair normal function. In principle, the ability to drive specific plasticity to reverse this pathological activity could renormalize circuits and restore function [1]. Rehabilitative therapies aim, in part, to facilitate training-dependent adaptive plasticity to support recovery of normal function. However, traditional rehabilitation following serious neurological injury or disease often yields only modest improvement and leaves many patients with chronic disability. The development of adjunctive strategies that broadly support plasticity to facilitate the benefits of rehabilitation has the potential to improve outcomes for a wide range of neurological disorders. Recently, a novel targeted plasticity therapy using brief bursts of vagus nerve stimulation (VNS) paired with rehabilitative training has emerged as a potential flexible method to enhance the benefits of rehabilitative interventions $[2,3]$.

Interest in the vagus nerve as a means to facilitate plasticity stems from early studies that implicated the nerve in enhanced consolidation of memory. In rodents, behavioral training immediately followed by administration of a variety of peripherally acting agents that do not readily cross the blood-brain barrier, including cholecystokinin, bombesin, gastrinreleasing peptide, 4-OH amphetamine, substance $\mathrm{P}$, and Lglucose, improved consolidation of memory [4-9]. Vagotomy 
blocked the memory enhancing effects of these chemical stimuli, suggesting that the ascending projections of the vagus may act as a conduit to relay peripheral information to the central nervous system (CNS) [4-9]. If chemical stimulation with these agents activates fibers of the vagus nerve to enhance memory, then electrical stimulation of the nerve would be expected to have a similar effect. In a seminal experiment, Clark et al. [10] demonstrated that electrical stimulation of the vagus nerve immediately following training enhanced memory, providing a direct link between activity in the vagus nerve and modulation of CNS function [10].

The anatomical and functional connectivity of the vagus nerve provides a clear basis for its effects on the CNS. While most often recognized for its descending projections to viscera, $>80 \%$ of the vagus nerve consists of afferent connections that terminate in the nucleus tractus solitarius in the brainstem [11-14]. Electrical stimulation of the vagus nerve drives activity in the cholinergic basal forebrain and the noradrenergic locus coeruleus, and results in subsequent release of neuromodulators throughout the cortex [15-20]. A reduction in either noradrenergic or cholinergic transmission blocks the effects of VNS in the CNS [21, 22]. Both of these neuromodulatory systems are key substrates in the expression of cortical plasticity [23-31], providing a rationale by which VNS paired with rehabilitation could enhance recovery. The succeeding sections detail studies that evaluate the ability of targeted plasticity therapies using VNS paired with rehabilitative training to drive diverse, long-lasting plasticity and improve recovery in the context of neurological injury and disease.

\section{Enhancing Plasticity with VNS}

\section{VNS Paired with Tones Drives Reorganization in the Auditory Cortex}

In an early investigation of the ability of VNS to enhance cortical plasticity, Engineer et al. [32] examined whether left cervical VNS paired with the presentation of tones could drive reorganization of the tonotopic map in primary auditory cortex (Fig. 1A). Rats were repeatedly presented with a midfrequency pure tone over the course of 20 days either with or without paired VNS, followed by auditory mapping to derive the frequency map in primary auditory cortex. Consistent with previous studies, repeated presentation of tones without VNS did not induce map reorganization [33]. However, presentation of the same number of tones paired with VNS significantly increased the proportion of neurons that responded to frequencies near the paired tone. A second cohort of rats was repeatedly presented with a high-frequency tone with or without paired VNS. Similar map expansion was observed corresponding to the paired high frequency tone with no change in mid-frequency area in rats that received VNS, suggesting that the observed reorganization is due to the specific event paired with VNS rather than a generalized increase in excitability in auditory cortex. Interleaved low-frequency tones that were not paired with VNS did not exhibit an increased response, suggesting VNS can label temporally specific stimuli. In addition to the changes in spectral response properties, VNS can also enhance temporal response characteristics of neurons in the primary auditory cortex [34]. Presentation of rapid trains of tones paired with VNS increased the maximal following rate of neurons within primary auditory cortex compared with naïve controls, while presentation of slow trains of tones paired with VNS decreased maximal following rate. Additionally, VNS drives plasticity specific in auditory cortex to complex stimuli. VNS repeatedly paired with speech sounds increases neural response strength to the paired speech sounds but not to novel, unpaired speech sounds [35]. Together, these findings indicate that VNS repeatedly paired with various auditory training regimens can drive specific changes in multiple characteristics of neuronal responses in the auditory cortex. The potential mechanisms that support VNS-dependent plasticity are discussed in detail below.

\section{VNS Paired with Forelimb Training Enhances Reorganization of Movement Representations in the Motor Cortex}

Based on the robust enhancement of auditory cortex plasticity, Porter et al. [36] evaluated whether VNS could enhance eventspecific plasticity within the motor system (Fig. 1B). To do so, rats were trained to perform 1 of 2 skilled motor tasks. The first task was designed to engage primarily the upper forelimb and required the rat to press rapidly a lever located outside the cage. The second task was designed to engage mainly the paw and required the rat to reach through a narrow slot in the floor of the cage and spin a small wheel with the digits and wrist. Once proficient on one of the tasks, rats underwent 5 days of training with or without left cervical VNS delivered on successful trials. On the day following the last training session, intracortical microstimulation mapping was used to document movement representations in motor cortex. Rats that received repeated VNS paired with training on the lever-press task demonstrated a considerable increase in size of the upper forelimb representation but no change in paw representation compared with rats that did not receive VNS. Similarly, rats that received VNS paired with training on the wheel-spin task exhibited a significant increase in the area of motor cortex representing the paw with no expansion of upper forelimb representation. These findings demonstrate that VNS facilitated robust expansion of the motor cortex representation of the specific movement that was paired with stimulation. This parallels the results observed in the auditory cortex and indicates 
Tone presentation

A
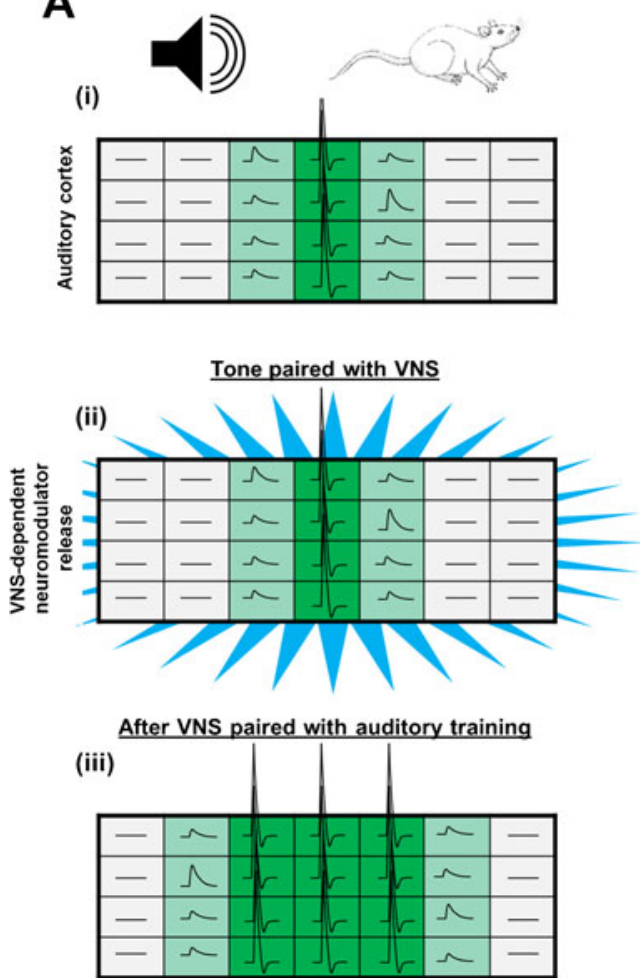

Fig. 1 Vagus nerve stimulation (VNS)-dependent enhancement of plasticity in cortical circuits. (A) (i) Presentation of a tone drives activity in the auditory cortex (green). (ii) Temporally precise release of neuromodulators (blue), such as that induced by VNS, paired with this activity drives plasticity. (iii) After repeated tone presentation paired with VNS, map reorganization results in an increased representation of the paired tone [32]. Previously subthreshold inputs (light green) drive activity after pairing with VNS. (B) (i) Activity within neurons of the motor cortex results in movement of the shoulder. (ii) Release of

that VNS paired with events can induce robust plasticity specific to the paired event.

The neural mechanisms that underlie VNS-dependent enhancement of plasticity are unknown. VNS drives activity in the cholinergic basal forebrain, providing a potential pathway to enhance plasticity $[15,33]$. Our recent study directly tested whether the nucleus basalis (NB), a primary component of the cholinergic basal forebrain, is required for VNS-dependent enhancement of map plasticity in motor cortex. Rats were trained to perform the lever-pressing task emphasizing upper forelimb described above [36, 37]. Once proficient, rats received an immunotoxin lesion that specifically depletes cortical cholinergic innervation from the NB or a sham lesion, followed by 5 days of training with VNS paired with successful trials. In rats with an intact NB, VNS paired with motor training significantly increased the representation of the upper forelimb in motor cortex. NB lesions prevented the VNSdependent increase in upper forelimb representation, indicating that the cortical cholinergic projections from the NB are required for VNS-dependent plasticity. While this study is
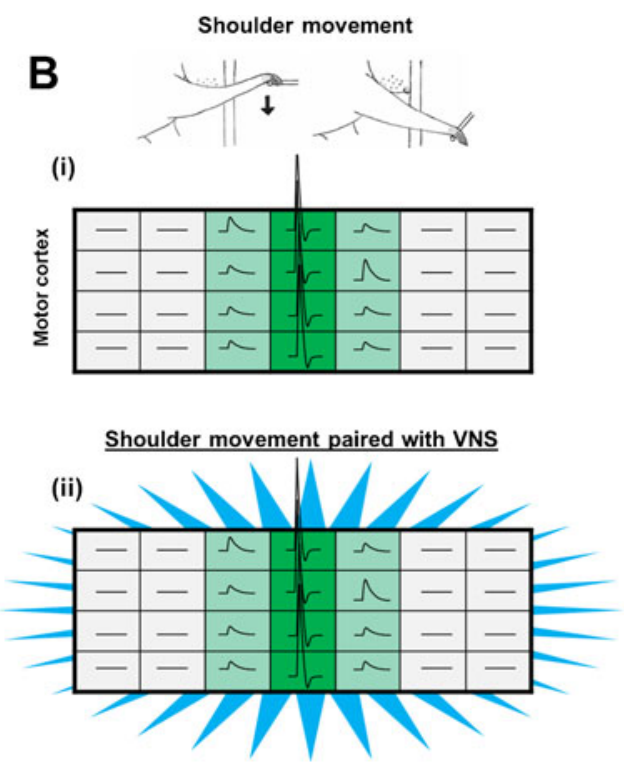

(iii)

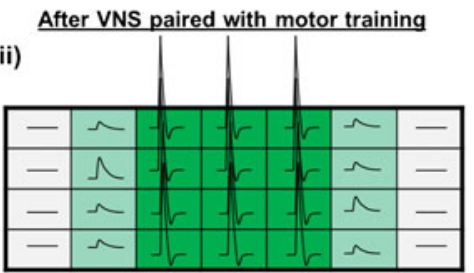

neuromodulators paired with movement drives plasticity in the motor cortex. (iii) Following repetitive shoulder movement paired with VNS, the number of circuits representing shoulder movement in the motor cortex is increased [36]. The large rectangles represent topographical organization of the auditory and motor cortices, and the activity of neurons is represented within each individual box. Dark green denotes suprathreshold action potential firing, light green denotes subthreshold depolarization, and gray denotes no response. Figure adapted from [3]

restricted to motor cortex, engagement of cholinergic circuitry may represent a convergent mechanism that underlies the plasticity-enhancing effects of VNS.

\section{Stimulation of the Vagus Nerve During a Cognitive Task Enhances Memory}

In addition to the topographical manifestations of cortical plasticity, delivery of VNS after behavioral experience can enhance memory retention. In a pioneering study, Clark et al. [10] investigated whether stimulation of the vagus nerve after inhibitory avoidance training could improve consolidation of avoidance memories. Rats were trained on a single trial inhibitory avoidance task, followed immediately by a 30 -s train of VNS or no stimulation. Upon retest $24 \mathrm{~h}$ later, rats that received VNS demonstrated a remarkable increase in retention compared with rats that did not receive stimulation. Inactivation of vagal fibers below the point of stimulation did not prevent increased retention, suggesting that VNS enhances memory retention through direct ascending inputs into 
the CNS [38]. To extend these findings, the authors conducted a follow-up study to evaluate the ability of VNS to enhance memory in humans [39]. Subjects read paragraphs with a subset of highlighted words and were given VNS or sham stimulation immediately after reading. In a recognition memory test, VNS significantly improved the subjects' ability to recognize previously highlighted words in a list of distracters. These findings provide support for the ability of VNS paired with experience to enhance memory retention.

\section{Using VNS to Enhance Rehabilitative Therapy}

As VNS has the ability to enhance specifically plasticity when delivered during different modalities of training, it has garnered interest as a potential targeted plasticity therapy to boost the benefits of a variety of distinct rehabilitative training regimens. In the sections below, we outline the preclinical and clinical studies that use VNS to enhance the benefits of rehabilitation and promote recovery in a variety of sensory, motor, and cognitive disorders.

\section{Targeted Plasticity Therapy to Treat Sensory Disorders}

\section{Preclinical and Clinical Studies for Tinnitus}

Tinnitus, or the perception of sound when no sound is present, is a common disorder that can cause minor irritation to devastating reduction in quality of life [40, 41]. Current treatments are largely ineffective, with great variability in patient response and risk for significant adverse effects [41, 42]. While the pathophysiology is still unclear, it is generally accepted that maladaptive plasticity within auditory circuitry of the CNS is at least, in part, responsible for chronic tinnitus [43]. In response to noise-induced hearing loss, the CNS fails to receive input from a region of the damaged cochlea. This loss of input causes destabilization of the normal excitatory and inhibitory balance within central auditory circuits, which can lead to map distortion, increased receptive field size, and increased synchronous activity in quiet, all of which appear to contribute to the tinnitus percept [32].

As detailed above, VNS paired with tones can drive specific plasticity to alter spectral and temporal response characteristics of central auditory neurons [32, 34]. If map distortion and alterations in receptive field size contribute to tinnitus, in principle, VNS paired with the appropriate presentation of tones could drive plasticity to restore the normal characteristics of the circuitry and alleviate the percept of tinnitus (Fig. 2A). Engineer et al. [32] sought to evaluate the ability of VNS-based targeted plasticity therapy to eliminate the behavioral correlate of chronic tinnitus in rats. The rationale for the therapy was based on increasing the number of cortical neurons tuned to frequencies other than the tinnitus frequency to reduce the overrepresented tinnitus frequency. Noise trauma was induced to damage the cochlea, causing a substantial increase in the proportion of neurons tuned to middle frequency tones, a reduction in the proportion of neurons responding to high frequency tones, and an increase in overall synchrony, all reflective of changes proposed to be responsible for tinnitus. Rats displaying a tinnitus percept centered on middle frequency tones were assigned to receive either VNS tone therapy or sham therapy over the course of 4 weeks. The VNStone therapy consisted of left cervical VNS paired with randomly interleaved tones that spanned the rat hearing range but excluded the tinnitus frequencies. VNS tone therapy fully ameliorated the tinnitus percept within 2 weeks of the initiation of therapy. Reflective of a reversal of tinnitus with VNS, rats that received VNS paired with tone therapy were able to detect a gap in a tone centered on the tinnitus frequency significantly better than rats that received sham therapy. These behavioral improvements were observed for up to 3 months after the end of VNS tone therapy, suggesting that the effects of the therapy were persistent. Sham therapy failed to improve the tinnitus percept at any of time during therapy. Correlating with the behavioral improvements, VNS tone therapy reversed many electrophysiological correlates of tinnitus in auditory circuitry, including map distortion and elevated synchrony. This study provides evidence that VNS paired with tones can reverse pathological plasticity and ameliorate chronic tinnitus.

Based on these preclinical findings, VNS paired with tones was investigated in an open-label trial in patients with severe chronic tinnitus [44-46]. Patients received left cervical VNS paired with a range of tones, excluding the tinnitus frequency, for $2.5 \mathrm{~h}$ each day for 20 days. At the conclusion of therapy, $4 / 10$ patients demonstrated clinically significant improvements in qualitative inventories of handicap and quantitative measures of minimum masking level. These benefits persisted 2 months after the conclusion of therapy, suggesting that the therapy may promote lasting reduction of symptoms. The persistence of therapeutic benefits is consistent with a VNS-dependent reversal of maladaptive plasticity in auditory circuitry rather than a nonspecific effect of stimulation. Of the 6 patients that did not respond to the therapy, 5 were on medications that interfered with neuromodulatory transmission. The absence of benefits in these patients aligns with the hypothesized role of the cholinergic and noradrenergic systems as key substrates in VNS-based targeted plasticity therapies. In practical terms, these findings draw attention to the need to delineate clearly the neuromodulatory mechanisms that are engaged by VNS in order to identify pharmacological and comorbid conditions that may occlude the benefits of the therapy. A recent placebocontrolled case study provides further support for the efficacy of VNS therapy to treat chronic tinnitus [47]. 

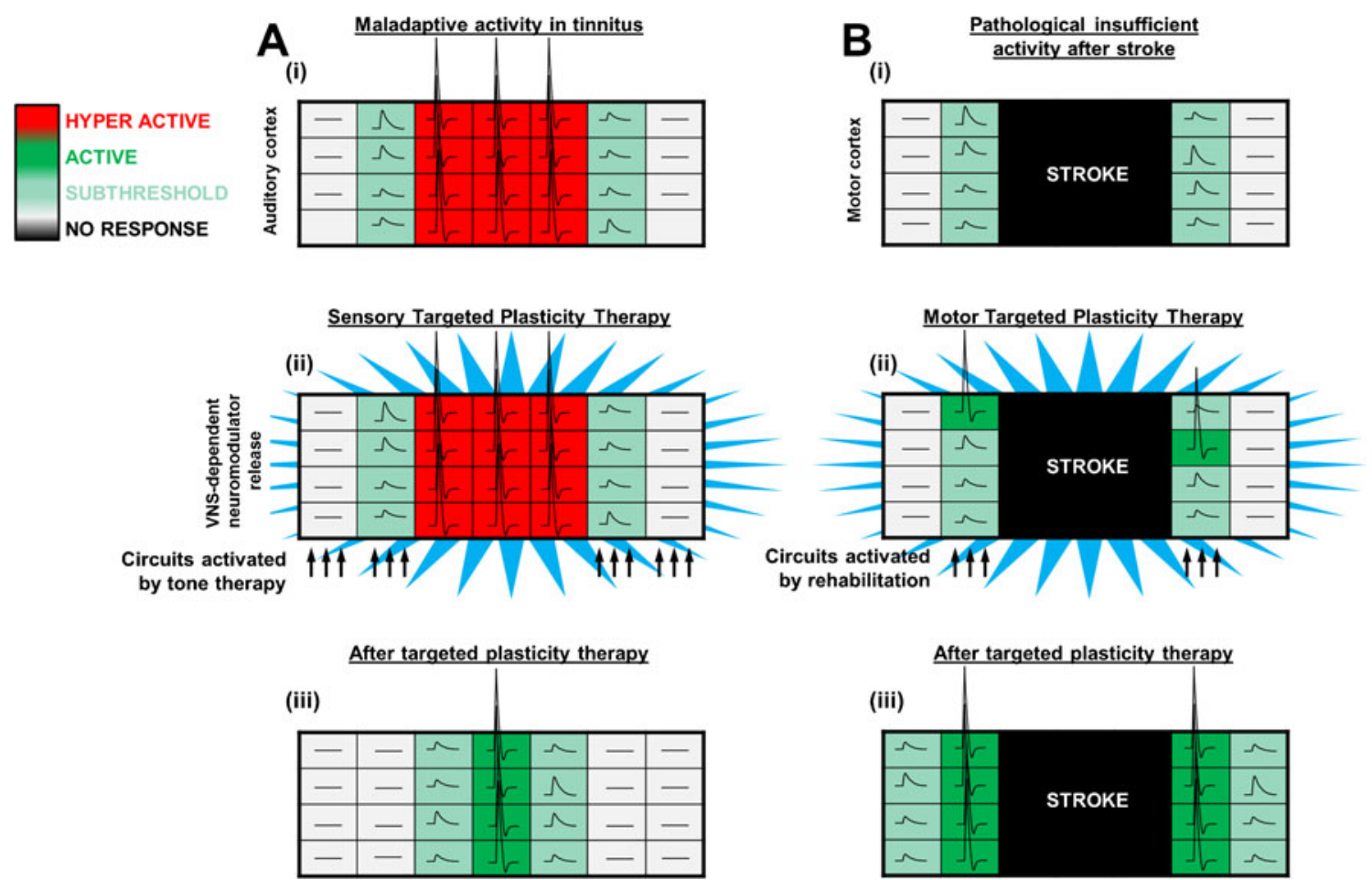

Fig. 2 Model of vagus nerve stimulation (VNS)-based targeted plasticity therapies. (A) (i) In tinnitus, auditory neurons are hyperactive and the tonotopic map is distorted. (ii) Presentation of tones excluding the tinnitus frequency paired with VNS drives adaptive plasticity within the auditory system. (iii) Activity within the auditory system is renormalized after targeted plasticity therapy, demonstrating that VNS paired with sensory rehabilitation can reverse maladaptive plasticity [32]. (B) (i)

Together, these results indicate VNS-based targeted plasticity therapies hold promise to treat tinnitus. However, a larger, placebo-controlled, double-blind study is needed to provide a thorough investigation of the efficacy of VNS therapy in the broader clinical population of patients with tinnitus.

\section{Potential Applications for Other Sensory Disorders}

Targeted plasticity therapies utilizing VNS have the potential to treat a wide variety of sensory disorders, depending on the rehabilitative regimen paired with stimulation. Maladaptive plasticity in the CNS is believed to contribute to the percept phantom limb pain and some forms of chronic pain. In amputees, cortical reorganization can be accompanied by phantom limb pain, which is correlated with the degree of overrepresentation [48]. Some forms of chronic pain unrelated to limb loss display a similar manifestation of aberrant cortical plasticity $[49,50]$. Similar to the implementation of targeted plasticity therapy for tinnitus, pairing VNS with various modalities of cutaneous somatosensory stimulation excluding the overrepresented painful area may potentially reverse associated maladaptive plasticity and reduce the percept of pain.
Following a stroke, circuits controlling movement are damaged resulting in impaired function. (ii) Motor rehabilitation paired with VNS drives robust neural plasticity in motor cortex. (iii) After targeted plasticity therapy, the number of circuits controlling the previously impaired movement are increased, leading to enhanced recovery of motor function [59-61, 66]. Figure adapted from [3]

\section{Targeted Plasticity Therapy to Treat Motor Disorders}

\section{Preclinical and Clinical Studies for Ischemic Stroke}

Ischemic stroke is a leading cause of disability, affecting 795 , 000 people in the USA each year [51]. As many as $75 \%$ of cases lead to lasting impairments in upper limb function [52]. Physical rehabilitation is the most common post-troke intervention. Rehabilitation can yield some functional gains, but recovery is often incomplete and the majority of patients are left with chronic disability [52-54]. Motor networks in surviving peri-infarct regions, as well as the undamaged contralateral homotopic cortex and subcortical structures, demonstrate significant reorganization after stroke [55-57]. Plasticity in these areas is believed to support functional recovery [58]. As detailed above, a study from Porter et al. [36] demonstrated that VNS paired with forelimb training drives robust, specific plasticity within motor cortex. Based on this enhancement of plasticity, VNS paired with rehabilitative training represents a potential method to enhance reorganization within spared circuitry after stroke and improve recovery of motor function (Fig. 2B).

Khodaparast et al. [59] tested this hypothesis using a rat model of ischemic stroke. In an initial study, rats were trained 
on the bradykinesia assessment task, a quantitative measure forelimb movement speed [37]. Once proficient, rats received a cortical ischemic lesion that impaired function of the trained limb. Rats were then assigned to receive rehabilitative training with or without the delivery of VNS on successful trials. VNS was delivered during rehabilitative sessions over the course of 5 weeks. VNS paired with rehabilitative training fully restored forelimb performance by the second week of treatment and significantly improved recovery compared to rehabilitative training without VNS. A second study by the same group extended these findings to recovery of forelimb strength after stroke [60]. VNS paired with rehabilitative training resulted in significantly greater recovery of volitional forelimb strength over the course of therapy compared with rehabilitative training without VNS. These benefits persisted after the cessation of VNS, suggesting a long-term improvement. Moreover, $100 \%$ of subjects that received VNS paired with rehabilitative training demonstrated a full recovery of forelimb strength, while only $22 \%$ of subjects that received rehabilitative training without VNS demonstrated a full recovery. In both studies, no difference in lesion size was observed, suggesting that VNS is not conferring a neuroprotective effect but rather improving recovery by enhancing plasticity. A follow-up study provided additional evidence that neuroplasticity underlies the benefits of VNS therapy for stroke [61]. A matched amount of VNS that is not paired with rehabilitative training is less effective in restoring function than VNS that is paired with rehabilitative training, suggesting that nonspecific effects of stimulation are unlikely to underlie VNS-dependent enhancement recovery.

Rehabilitative strategies have the highest potential to improve functional recovery after stroke when delivered early, and efficacy diminishes substantially with increasing time after stroke [62-65]. A study conducted by Khodaparast et al. [66] sought to evaluate whether VNS paired with rehabilitative training would be effective after a long delay between ischemic lesion and therapy initiation. To this end, rats were trained to proficiency on the isometric force task and received an ischemic lesion of the motor cortex and dorsolateral striatum to impair use of the trained forelimb. Beginning 7 weeks after lesion, rats received either VNS paired with rehabilitative training, equivalent rehabilitative training with a matched amount of VNS delivered $2 \mathrm{~h}$ after daily training sessions, or rehabilitative training without VNS. Even when initiated many weeks after stroke in rats with stable, chronic deficits, VNS paired with rehabilitative training significantly enhanced recovery of forelimb function compared with rehabilitative training without VNS. Consistent with previous studies, a matched amount of VNS that was not paired with rehabilitative training failed to improve recovery [61]. In summary, a number of preclinical studies provide proof-of-concept demonstration of efficacy for VNS paired with rehabilitative training to improve recovery of motor function after ischemic stroke.
Based on these studies, Dawson et al. [67, 68] evaluated VNS paired with physical rehabilitation in a pilot study in stroke patients. Although designed as a safety study, VNS was paired with several typical rehabilitative tasks over the course of 6 weeks of physical rehabilitation. At the conclusion of therapy, patients who received VNS paired with rehabilitation demonstrated a significant 3-fold increase in change of Upper Extremity Fugl-Meyer score compared with patients who received the same rehabilitation without VNS. The improved forelimb function persisted when evaluated 30 days after the conclusion of therapy, potentially suggesting a longlasting recovery of function. While these findings are promising, an ongoing well-controlled clinical trial will provide an additional evaluation of clinical efficacy in stroke patients [69].

\section{Preclinical Studies for Intracerebral Hemorrhage}

Intracerebral hemorrhage (ICH) is a devastating subtype of stroke, affecting as many as 52,000 people each year in the USA [70]. ICH has a mechanistically distinct pathophysiology from ischemic stroke, characterized by bleeding in the brain parenchyma, which often damages cortical and subcortical structures [70]. A recent global epidemiological study indicates that the disability adjusted life years lost to ICH is nearly double that lost to ischemic stroke, highlighting the significant burden of this disease [71]. Like ischemic stroke, plasticity within spared circuitry is believed to support recovery after ICH [72-74]. Therefore, VNS paired with rehabilitative training may enhance reorganization within these motor circuits and support recovery. Alternatively, the extensive damage to subcortical structures and white matter may prevent the beneficial effects of VNS therapy.

Our group evaluated whether VNS paired with rehabilitative training could improve recovery of forelimb function in a rat model of ICH [75]. Rats were trained to proficiency on a skilled forelimb task and then received a severe hemorrhagic lesion in the midbrain to impair use of the trained forelimb. Following lesion, rats underwent 6 weeks of rehabilitative training either with or without VNS. VNS paired with rehabilitative training resulted in significantly improved forelimb function compared to rehabilitative training without VNS. However, VNS therapy failed to restore fully forelimb function to prelesion levels, unlike the complete recovery observed in models of ischemic stroke [59-61]. To determine whether recovery was limited by an insufficient amount of VNS, a cohort of rats received VNS delivered on every attempted trial rather than only successful trials, resulting in $\sim 40 \%$ more paired stimulations during therapy. VNS paired with every trial on the rehabilitative task improved recovery compared with rehabilitative training alone, but did not result in a greater recovery compared with VNS paired with only successful trials. It is not clear whether the severity of the ICH lesion prevents full recovery, or whether further optimization of 
stimulation parameters or rehabilitative regimen may promote greater recovery. Regardless, these findings demonstrate that VNS paired with rehabilitative training improves recovery of forelimb function after severe striatal ICH.

\section{Preclinical Studies for Traumatic Brain Injury}

Traumatic brain injury (TBI) is a very common form of neurotrauma, with approximately 1.7 million annual cases in the USA [76]. The effects of TBI are by nature heterogeneous, but moderate-to-severe TBIs can result in chronic deficits in motor function [77, 78]. Recovery of motor function is associated with plasticity in spared motor circuits [79-81], and VNS paired with rehabilitation may enhance this plasticity to support recovery. Pruitt et al. [82] evaluated whether VNS therapy could improve recovery of motor function in a controlled cortical impact model of severe TBI. Rats were trained to proficiency on a skilled forelimb task and then received a controlled cortical impact centered on the motor cortex. VNS paired with rehabilitative training significantly improved recovery of volitional forelimb strength compared with rehabilitative training without VNS after TBI. Consistent with previous studies, VNS did not reduce lesion size, suggesting that VNS does not improve motor recovery through gross neuroprotection $[59,60,75]$. These findings extend the efficacy of VNS paired with rehabilitative training to a model of TBI.

\section{Potential Applications for Other Motor Disorders}

The proof-of-concept demonstration of efficacy in distinct models of ischemic stroke, ICH, and TBI exemplifies the flexibility of VNS-based targeted plasticity therapy and indicates that the benefits are not limited to a single mechanism of neurotrauma. Injuries to the spinal cord and peripheral nerves cause significant motor dysfunction and recovery is limited, in part, by insufficient and maladaptive plasticity in motor circuits [83-85]. Thus, a similar implementation of VNS paired with rehabilitative training may be effective for these injuries. Alternatively, the extensive white matter damage associated with the injuries may prevent the benefits of VNS therapy. Parkinson disease (PD) is another neuromotor disease associated with maladaptive plasticity in motor networks that could potentially be improved by VNS therapy [86-89]. However, alterations in neuromodulatory function associated with PD, particularly those resulting from neuronal death in the locus coeruleus, may occlude benefits of the therapy [90, 91]. Moreover, without abatement of progressive neuronal death, targeted plasticity therapy may offer little benefit for PD. Additional preclinical studies are needed to evaluate VNS therapy in the context of these injuries and diseases, as well as models that incorporate other clinically relevant complicating factors such as aging that may interfere with the benefits of the therapy.

\section{Targeted Plasticity Therapy to Treat Cognitive and Psychiatric Disorders}

\section{Preclinical Studies for Post-traumatic Stress Disorder}

Post-traumatic stress disorder (PTSD) is a prevalent, debilitating, treatment-resistant psychiatric disorder [92]. Aberrant plasticity is believed to underlie the hypersensitivity and abnormal memory retention that accompanies PTSD [93, 94], and reversal of this maladaptive plasticity may reduce the exaggerated fear response [95]. Prolonged exposure therapy, which involves repeated recall of an anxiety-inducing situation in a safe environment, provides a degree of desensitization that reduces the fearful response [96, 97]. VNS may represent an adjunctive strategy to potentiate the effects of exposure therapy to normalize the hypersensitive responses and improve the symptoms of PTSD [98].

A proof-of-principle study conducted by Peña et al. [94] in a rat model of PTSD lends credence to this hypothesis. Rats were trained on an auditory fear conditioning task followed by 1 session of extinction training with or without VNS. Conditioned fear retention was assessed 1 day later. VNS paired with extinction training resulted in a significant reduction of conditioned fear compared with extinction training without VNS. Conditioned fear remained reduced 2 weeks after the cessation of stimulation, suggesting that the beneficial effects of VNS may be long-lasting. Additionally, VNS paired with extinction training was similarly effective at reducing a remote fear memory compared to extinction training without VNS. Unpaired VNS delivered shortly after training failed to reduce conditioned fear retention, suggesting that VNS must be temporally paired with exposure to enhance fear reduction. Although chronic VNS is known to confer anxiolytic effects [99, $100]$, this effect is not dependent on pairing with an extinction training regimen. Therefore, the absence of benefits of unpaired VNS is consistent with VNS therapies for sensory and motor dysfunction described above [32, 59, 61], and further supports enhancement of plasticity as a convergent mechanism that underlies the benefits of VNS therapy.

A follow-up study provides direct evidence of VNSdependent alterations in synaptic plasticity that accompany extinction training [101]. The infralimbic medial prefrontal cortex (IL) and basolateral amygdala (BLA) are implicated in acquisition of conditioned fear responses [102]. Moreover, plasticity in these regions is believed to mediate reversal of conditioned fear responses with extinction [103, 104]. Peña et al. [101] hypothesized that VNS may alter the strength of connections between these areas to enhance the extinction of fear. Rats were trained on an auditory avoidance task followed by 1 day of extinction training with or without paired VNS. Replicating the findings of the previous study, extinction training paired with VNS results in enhanced extinction of fear behavior compared with extinction training alone [94]. At 
the completion of behavioral testing, strength of the connection from the IL to the BLA was measured before and after a short train of burst stimulation in the IL. VNS and extinction training had complex, interacting effects on the plasticity of IL-BLA connections. Burst stimulation of the IL evoked longterm potentiation in the IL-BLA projections in rats that received VNS paired with extinction training. In contrast, burst stimulation evoked long-term depression in rats that received extinction training without VNS. Naïve rats and rats that received VNS without extinction training failed to show plasticity in IL-BLA connection strength after burst stimulation. These findings suggest that VNS paired with extinction training may promote plasticity in the IL-BLA pathway to support reduction of conditioned fear.

\section{Potential Applications for Other Cognitive and Psychiatric Disorders}

Maladaptive plasticity is associated with a variety of cognitive and psychiatric disorders, including anxiety, bipolar disorder, schizophrenia, depression, drug addiction, and attentiondeficit hyperactivity disorder [2, 105]. The complexity of these disorders have left them significantly undermanaged or palliated with pharmaceuticals that carry a high risk of dependence and overdose, emphasizing the need for effective, flexible treatments that can address the underlying pathophysiology. VNS, if paired with the appropriate regimen of behavioral exposure or cognitive training, may represent a potential intervention to improve these disorders. Plasticity is a core deficit in many postnatal developmental disorders, including autism and forms of mental retardation [106-109]. Rehabilitative therapies do provide benefits in patients with postnatal developmental disorders. VNS delivered during these therapies may potentiate the benefits of rehabilitation and provide greater functional improvements [110-112]. However, if plasticity is altered, it is possible that targeted plasticity therapy may fail to benefit these patients. Cognitive disorders pose significant rehabilitative challenges owing both the complexity and heterogeneity, but the flexibility to pair VNS with virtually any rehabilitative regimen holds potential to treat at least some aspects of these disorders.

\section{Challenges for Translating VNS Therapies into Clinical Practice}

\section{Defining Factors that Limit Effectiveness of VNS}

Despite the promising preclinical and clinical evidence of VNS in a variety of neurological diseases and injury, significant challenges remain as targeted plasticity therapies using VNS are translated into clinical use. Common clinical factors that interfere with plasticity may occlude the effects of VNS.
For instance, advanced age is linked with reduced capacity for plasticity and is very common in the population of ischemic stroke patients [51, 113-117]. Therefore, it will be necessary to define whether advanced age affects the efficacy of VNS in the context of stroke, in order to determine the patient population most likely to respond to the therapy. Many common pharmaceuticals target the cholinergic and noradrenergic systems engaged by VNS. Indeed, drugs that affect these neuromodulators substantially reduce the benefits of VNS therapy in tinnitus patients [45], indicating that these neuromodulatory pathways are necessary for the effects of VNS. Several diseases affect cell number and function in these neuromodulatory systems, including Alzheimer disease, PD, chronic alcoholism, Down syndrome, TBI, and PTSD [118-126]. Comorbidity with these and other diseases that impair neuromodulatory function may limit the effects of VNS. However, as plasticity is reduced but not fully abrogated in these conditions, different stimulation paradigms may be particularly effective in patients with these complicating factors. The diversity of the clinical population and likelihood of comorbid conditions highlights the importance of delineating the neuromodulatory pathways and mechanisms that underlie VNS-dependent recovery.

\section{Optimizing VNS Parameters}

Determining the optimal stimulation parameters of VNS is needed to improve the benefits of targeted plasticity therapy for clinical implementation. The cervical level of the vagus nerve is comprised of myelinated A and B fibers, as well as unmyelinated $\mathrm{C}$ fibers $[127,128]$. There is considerabe diversity in morphological and functional parameters of the nerve from individual to individual, suggesting that optimal stimulation parameters may also vary $[129,130]$. Moreover, higher VNS intensities recruit more vagal fibers and trigger greater release of neuromodulators, which may improve efficacy [19, 131, 132]. However, VNS-dependent enhancement of memory retention is very sensitive to the intensity of stimulation. In animal models and humans, moderate intensity stimulation yields significantly greater memory retention compared with lower and higher intensity stimulation [10, 38, 39]. A recent report demonstrates a similar inverted-U relationship of VNS on map reorganization in auditory cortex [133]. In this study, rats received repeated presentations of a mid-frequency tone paired with different stimulation intensities of VNS over the course of 20 days. Moderate-intensity ( 0.4 and $0.8 \mathrm{~mA})$ stimulation resulted in significantly greater map plasticity than higher-intensity (1.2 and $1.6 \mathrm{~mA}$ ) stimulation. Taken together, these studies suggest that, independent of the experience paired with VNS, stimulation intensity has an inverted-U relationship with VNS-dependent plasticity.

The inverted-U relationship mirrors the Yerkes-Dodson law, relating arousal and performance [134]. Other 
peripherally acting agents that conduct ascending information through the vagus nerve demonstrate a similar inverted-U response $[4,5,135-138]$. The precise mechanisms that underlie this response profile are not understood, but several explanations could account for the inverted-U response. First, a lowthreshold positive process and an opposing high-threshold negative process with sigmoidal responses could yield the inverted-U shape. In such a system, moderate levels of intensity would drive the optimal response by maximizing positive activation and minimizing negative activation. In the context of VNS, moderate levels of stimulation may optimally engage excitatory circuitry to support plasticity, while inhibition dominates at higher stimulation intensities and occludes plasticity. Alternatively, a single desensitizing system could explain the inverted-U response. Moderate intensity stimulation would provide sufficient activation with minimal desensitization, while high levels of stimulation would result in overactivation and reduction of the response. G protein-coupled receptors, such as those which likely mediate the response to VNSdependent engagement of the cholinergic and noradrenergic systems, exhibit notable desensitization and may explain the inverted-U response to VNS $[139,140]$. While these models can account for the inverted-U, they are not mutually exclusive and various other systems likely contribute. This inverted-U shaped relationship poses challenges for clinical implementation of VNS therapies, as more stimulation does not necessarily translate to more efficacy. Additionally, different diseases may perturb the systems engaged by VNS, thus shifting the set of optimal parameters. The fact that VNS is largely effective across of a 2 -fold range of intensities suggests that a potentially wide therapeutic range exists. However, it would be valuable to identify a biomarker that reports VNSdependent activation that would allow individual tailoring of stimulation levels for each patient as targeted plasticity therapies using VNS are translated into clinical practice.

\section{Identifying Mechanisms that Underlie Recovery}

The mechanisms that underlie the benefits of VNS therapy are unclear, but VNS engages a variety of molecular and neuronal mechanisms that may support plasticity. VNS increases levels of brain-derived neurotrophic factor (BDNF), a key regulator of plasticity [17]. BDNF activates multiple downstream effector elements associated with synaptic plasticity, including cyclic adenosine monophosphate response element binding protein, activity-regulated cytoskeletal protein (Arc), and CaMKII [141-144]. VNS increases phosphorylation at multiple residues in the BDNF receptor, TrkB, at sites known to be linked to activation of phospholipase $\mathrm{C}-\gamma$, phosphatidylinositol-3 kinase, and mitogen-activated protein kinase, all of which can modulate plasticity [145-147]. Inhibition of Trk autophosphorylation prevents VNS-dependent phosphorylation, indicating that VNS activates Trk through canonical mechanisms
[147]. In addition to changes in molecular signaling pathways, VNS induces changes in synaptic and intrinsic neuronal properties that may support recovery. VNS-dependent release of acetylcholine and norepinephrine may act together to alter spike-timing dependent plasticity properties to facilitate plasticity in active networks $[148,149]$. Moreover, VNS may directly alter synaptic properties by regulating expression of $\mathrm{N}$ methyl-D-aspartate receptor and $\gamma$-aminobutyric acid receptor A [150]. Low-intensity VNS results in activation of a slow hyperpolarizing current in cortical neurons, suggesting that VNS also influences intrinsic neuronal properties [151]. Changes in neuronal activity manifest as changes in network properties, as VNS induces desynchronization of cortical networks and increases power in low-frequency EEG bands [22, 152]. The engagement of these diverse neuronal pathways by VNS provides a framework by which VNS modulates cellular signaling to drive plasticity. A clear delineation of the molecular mechanisms that underlie VNS-dependent enhancement of plasticity may facilitate rational development of targeted plasticity therapies.

\section{Concluding Remarks}

While the preceding discussion focuses largely on VNS, it is critical to recognize that the core component of targeted plasticity therapy is the paired rehabilitative intervention. Rehabilitation uses repeated practice or experience and patient engagement to drive adaptive plasticity in neural circuits to support recovery of normal function (Fig. 3A). Practice results in neural activity while motivation yields neuromodulatory release, and these components act synergistically to drive neural plasticity in active circuits (Fig. 3B). In many cases, rehabilitation engages these components and is sufficient to improve recovery. However, in the context of severe neurological injuries or diseases, rehabilitation alone may be insufficient to
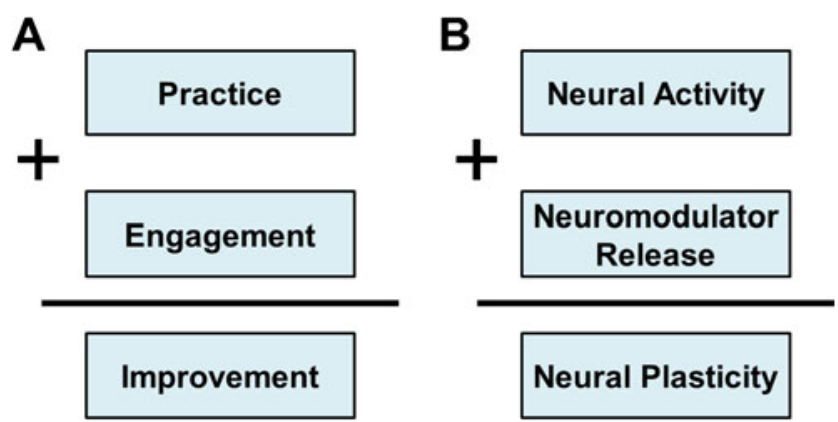

Fig. 3 Model of rehabilitation and neural plasticity. (A) During rehabilitation, repeated practice in conjunction with engagement (i.e., motivation and attention) yields improvements in function. (B) Neural activity coincident with neuromodulator release synergistically combine to drive neural plasticity. Targeted plasticity therapies provide precisely timed, nonadapting neuromodulator release paired with neural activity resulting from rehabilitation to facilitate plasticity and enhance recovery 
reverse pathological neural activity. In this context, targeted plasticity therapies act to provide repeated, nonadapting engagement of neuromodulatory systems to enhance rehabilitation-dependent plasticity. Thus, VNS acts to increase the gain, while rehabilitative training confers the specific neural changes that support recovery. Targeted plasticity therapies are not limited to the use of VNS to control neuromodulatory gain. In principle, other manipulations that can engage proplasticity pathways without directly interfering with ongoing activity in the rehabilitated circuits may act as an adjunctive strategy to boost the benefits of rehabilitation.

Targeted plasticity therapies have the potential to enhance a variety of rehabilitative training regimens and treat a range of neurological disorders and injuries. Preclinical studies and emerging evidence from pilot clinical trials demonstrate the potential efficacy and flexibility of using VNS therapy to improve recovery in sensory, motor, and cognitive neuropathologies. Future studies should focus on expanding the clinical indications for which VNS-based targeted plasticity therapies may offer benefits. Additionally, identifying clinically relevant factors that may occlude the benefits of VNS and optimizing delivery of the therapy are needed to facilitate clinical translation.

Acknowledgments I thank Dr. Michael Kilgard and Dr. Robert Rennaker for insightful comments and discussion about the manuscript. This work was supported by grants from the NIH National Institute for Neurological Disorders and Stroke (R01 NS085167), Defense Advanced Research Projects Agency (DARPA, ElectRx, HR0011-15-2-0017), and the Texas Biomedical Device Center.

\section{References}

1. Kilgard MP. Harnessing plasticity to understand learning and treat disease. Trends Neurosci 2012;35:715-722.

2. Lozano AM. Harnessing plasticity to reset dysfunctional neurons. N Engl J Med 2011;364:1367-1368.

3. Hays SA, Rennaker RL, II, Kilgard MP. Targeting plasticity with vagus nerve stimulation to treat neurological disease. Prog Brain Res 2013;207:275-299.

4. Flood JF, Smith GE, Morley JE. Modulation of memory processing by cholecystokinin: Dependence on the vagus nerve. Science 1987;236:832-834.

5. Flood JF, Morley JE. Effects of bombesin and gastrin-releasing peptide on memory processing. Brain Res 1988;460:314-322.

6. Williams C, Jensen RA. Effects of vagotomy on Leu-enkephalininduced changes in memory storage processes. Physiol Behav 1993;54:659-663.

7. Jensen RA. Modulation of memory storage processes by peripherally acting pharmacological agents. Proc West Pharmacol Soc 1996;39:85-89.

8. Talley CP, Clayborn H, Jewel E, McCarty R, Gold PE. Vagotomy attenuates effects of L-glucose but not of D-glucose on spontaneous alternation performance. Physiol Behav 2002;77:243-249.

9. Nogueira PJ, Tomaz C, Williams CL. Contribution of the vagus nerve in mediating the memory-facilitating effects of substance $\mathrm{P}$. Behav Brain Res 1994;62:165-169.
10. Clark K, Krahl S, Smith D, Jensen R. Post-training unilateral vagal stimulation enhances retention performance in the rat. Neurobiol Learn Mem 1994;63:213-216.

11. Foley JO, DuBois FS. Quantitative studies of the vagus nerve in the cat. I. The ratio of sensory to motor fibers. J Comp Neurol 1937;67:49-67.

12. Leslie R, Gwyn D, Hopkins D. The central distribution of the cervical vagus nerve and gastric afferent and efferent projections in the rat. Brain Res Bull 1982;8:37-43.

13. Berthoud H, Neuhuber WL. Functional and chemical anatomy of the afferent vagal system. Auton Neurosci 2000;85:1-17.

14. George MS, Sackeim HA, Rush AJ, et al. Vagus nerve stimulation: a new tool for brain research and therapy. Biol Psychiatry 2000;47:287-295.

15. Detari L, Juhasz G, Kukorelli T. Effect of stimulation of vagal and radial nerves on neuronal activity in the basal forebrain area of anaesthetized cats. Acta Physiol Hung 1983;61:147-154.

16. Dorr AE, Debonnel G. Effect of vagus nerve stimulation on serotonergic and noradrenergic transmission. J Pharmacol Exp Ther 2006;318:890-898.

17. Follesa P, Biggio F, Gorini G, et al. Vagus nerve stimulation increases norepinephrine concentration and the gene expression of BDNF and bFGF in the rat brain. Brain Res 2007;1179:28-34.

18. Groves DA, Bowman EM, Brown VJ. Recordings from the rat locus coeruleus during acute vagal nerve stimulation in the anaesthetised rat. Neurosci Lett 2005;379:174-179.

19. Roosevelt RW, Smith DC, Clough RW, Jensen RA, Browning RA. Increased extracellular concentrations of norepinephrine in cortex and hippocampus following vagus nerve stimulation in the rat. Brain Res 2006;1119:124-132.

20. Raedt R, Clinckers R, Mollet L, et al. Increased hippocampal noradrenaline is a biomarker for efficacy of vagus nerve stimulation in a limbic seizure model. J Neurochem 2011;117:461-469.

21. Krahl SE, Clark KB, Smith DC, Browning RA. Locus coeruleus lesions suppress the seizure-attenuating effects of vagus nerve stimulation. Epilepsia 1998;39:709-714.

22. Nichols J, Nichols A, Smirnakis S, Engineer N, Kilgard M, Atzori $M$. Vagus nerve stimulation modulates cortical synchrony and excitability through the activation of muscarinic receptors. Neuroscience 2011;189:207-214.

23. Gu Q. Neuromodulatory transmitter systems in the cortex and their role in cortical plasticity. Neuroscience 2002;111:815-835.

24. Sachdev RN, Lu S, Wiley RG, Ebner FF. Role of the basal forebrain cholinergic projection in somatosensory cortical plasticity. J Neurophysiol 1998;79:3216-3228.

25. Conner JM, Culberson A, Packowski C, Chiba AA, Tuszynski $\mathrm{MH}$. Lesions of the basal forebrain cholinergic system impair task acquisition and abolish cortical plasticity associated with motor skill learning. Neuron 2003;38:819-829.

26. Baskerville K, Schweitzer J, Herron P. Effects of cholinergic depletion on experience-dependent plasticity in the cortex of the rat. Neuroscience 1997;80:1159-1169.

27. Kasamatsu T, Shirokawa T. Involvement of $\beta$-adrenoreceptors in the shift of ocular dominance after monocular deprivation. Exp Brain Res 1985;59:507-514.

28. Kasamatsu T, Pettigrew JD, Ary M. Restoration of visual cortical plasticity by local microperfusion of norepinephrine. J Comp Neurol 1979;185:163-181.

29. Conner JM, Chiba AA, Tuszynski MH. The basal forebrain cholinergic system is essential for cortical plasticity and functional recovery following brain injury. Neuron 2005;46:173-179.

30. Ramanathan D, Tuszynski MH, Conner JM. The basal forebrain cholinergic system is required specifically for behaviorally mediated cortical map plasticity. J Neurosci 2009;29:5992-6000. 
31. Boyeson MG, Feeney DM. Intraventricular norepinephrine facilitates motor recovery following sensorimotor cortex injury. Pharmacol BiochemBehav 1990;35:497-501.

32. Engineer ND, Riley JR, Seale JD, et al. Reversing pathological neural activity using targeted plasticity. Nature 2011;470:101-104.

33. Kilgard MP, Merzenich MM. Cortical map reorganization enabled by nucleus basalis activity. Science 1998;279:1714-1718.

34. Shetake JA, Engineer ND, Vrana WA, Wolf JT, Kilgard MP. Pairing tone trains with vagus nerve stimulation induces temporal plasticity in auditory cortex. Exp Neurol 2011;233:342-349.

35. Engineer CT, Engineer ND, Riley JR, Seale JD, Kilgard MP. Pairing speech sounds with vagus nerve stimulation drives stimulus-specific cortical plasticity. Brain Stimul 2015;8:637-644.

36. Porter BA, Khodaparast N, Fayyaz T, et al. Repeatedly pairing vagus nerve stimulation with a movement reorganizes primary motor cortex. Cereb Cortex 2011;22:2365-2374.

37. Hays SA, Khodaparast N, Sloan AM, et al. The bradykinesia assessment task: an automated method to measure forelimb speed in rodents. J Neurosci Methods 2013;214:52-61.

38. Clark K, Smith D, Hassert D, Browning R, Naritoku D, Jensen R, Posttraining electrical stimulation of vagal afferents with concomitant vagal efferent inactivation enhances memory storage processes in the rat. Neurobiol Learn Mem 1998;70:364-373.

39. Clark KB, Naritoku DK, Smith DC, Browning RA, Jensen RA. Enhanced recognition memory following vagus nerve stimulation in human subjects. Nat Neurosci 1999;2:94-98.

40. Davis A, El Rafaie A. Epidemiology of tinnitus. Tinnitus handbook. Thomson Learning, San Diego, CA, 2000, pp. 1-23.

41. Lockwood AH, Salvi RJ, Burkard RF. Tinnitus. N Engl J Med 2002;347:904-910.

42. Parnes S. Current concepts in the clinical management of patients with tinnitus. Eur Arch Otorhinolaryngol 1997;254:406-409.

43. Eggermont JJ, Roberts LE. The neuroscience of tinnitus. Trends Neurosci 2004;27:676-682.

44. Microtransponder. Proof-of-concept study assessing VNS paired with tones for tinnitus. NCT01253616, Available at: http://clinicaltrials. gov/ct2/show/NCT01253616. Accessed August 20, 2015.

45. De Ridder D, Vanneste S, Engineer ND, Kilgard MP. Safety and efficacy of vagus nerve stimulation paired with tones for the treatment of tinnitus: a case series. Neuromodulation 2014;17:170179 .

46. Microtransponder. Vagus nerve stimulation (VNS) paired with tones for tinnitus. NCT01962558. Available at: http://clinicaltrials.gov/ ct2/show/NCT01962558. Accessed August 20, 2015.

47. De Ridder D, Kilgard M, Engineer N, Vanneste S. Placebocontrolled vagus nerve stimulation paired with tones in a patient with refractory tinnitus: a case report. Otol Neurotol 2015;36:575580

48. Flor H, Elbert T, Knecht $\mathrm{S}$, et al. Phantom-limb pain as a perceptual correlate of cortical reorganization following arm amputation. Nature 1995;375:482-484.

49. Birbaumer N, Lutzenberger W, Montoya P, et al. Effects of regional anesthesia on phantom limb pain are mirrored in changes in cortical reorganization. J Neurosci 1997;17:5503-5508.

50. Flor H. Cortical reorganisation and chronic pain: implications for rehabilitation. J Rehabil Med 2003;41(Suppl.):66-72.

51. Roger VL, Go AS, Lloyd-Jones DM, et al. Heart disease and stroke statistics - 2012 update a report from the American Heart Association. Circulation 2012;125:e2-e220.

52. Dobkin BH. Strategies for stroke rehabilitation. Lancet Neurol 2004;3:528-536.

53. Dobkin BH. Rehabilitation after stroke. N Engl J Med 2005;352: 1677-1684.

54. Lai S, Studenski S, Duncan PW, Perera S. Persisting consequences of stroke measured by the Stroke Impact Scale. Stroke 2002;33: $1840-1844$
55. Calautti C, Baron J. Functional neuroimaging studies of motor recovery after stroke in adults a review. Stroke 2003;34:15531566.

56. Nudo R, Friel K. Cortical plasticity after stroke: implications for rehabilitation. Rev Neurol 1999;155:713.

57. Zhang J, Meng L, Qin W, Liu N, Shi FD, Yu C. Structural damage and functional reorganization in ipsilesional $\mathrm{m} 1$ in well-recovered patients with subcortical stroke. Stroke 2014;45:788-793.

58. Hallett M. Plasticity of the human motor cortex and recovery from stroke. Brain Res Rev 2001;36:169-174.

59. Khodaparast N, Hays SA, Sloan AM, et al. Vagus nerve stimulation delivered during motor rehabilitation improves recovery in a rat model of stroke. Neurorehabil Neural Repair 2014;28:698-706.

60. Khodaparast N, Hays SA, Sloan AM, et al. Vagus nerve stimulation during rehabilitative training improves forelimb strength following ischemic stroke. Neurobiol. Dis 2013;60:80-88.

61. Hays SA, Khodaparast N, Ruiz A, et al. The timing and amount of vagus nerve stimulation during rehabilitative training affect poststroke recovery of forelimb strength. NeuroReport 2014;25:676-682.

62. Kwakkel G, Kollen BK, van der Grond J, Prevo AJH. Probability of Regaining dexterity in the flaccid upper limb impact of severity of paresis and time since onset in acute stroke. Stroke 2003;34: 2181-2186.

63. Biernaskie J, Chernenko G, Corbett D. Efficacy of rehabilitative experience declines with time after focal ischemic brain injury. J Neurosci 2004;24:1245-1254.

64. Teasell R, Bitensky J, Salter K, Bayona NA. The role of timing and intensity of rehabilitation therapies. Top Stroke Rehabil 2005; $12: 46$.

65. Katherine Salter B, Mark Hartley B, Norine Foley B. Impact of early vs delayed admission to rehabilitation on functional outcomes in persons with stroke. J Rehabil Med 2006;38:113-117.

66. Khodaparast N, Kilgard MP, Casavant R, et al. Vagus nerve stimulation during rehabilitative training improves forelimb recovery after chronic ischemic stroke in rats. Neurorehabil Neural Repair 2015 Nov 4 [Epub ahead of print].

67. Dawson J, Dixit A, Pierce D, et al. Safety, feasibility and efficacy of vagus nerve stimulation paired with upper limb rehabilitation following ischaemic stroke. Stroke (in press).

68. Microtransponder. Paired vagus nerve stimulation (VNS) with rehabilitation for upper limb function improvement after stroke. NCT01669161. Available at: https://clinicaltrials.gov/ct2/show/ NCT01669161. Accessed August 20, 2015.

69. Microtransponder. VNS during rehabilitation for improved upper limb motor function after stroke. NCT02243020. Available at: https://clinicaltrials.gov/ct2/show/study/NCT02243020. Accessed August 20, 2015.

70. Qureshi AI, Tuhrim S, Broderick JP, Batjer HH, Hondo H, Hanley DF. Spontaneous intracerebral hemorrhage. N Engl J Med 2001;344:1450-1460.

71. Krishnamurthi RV, Feigin VL, Forouzanfar MH, et al. Global and regional burden of first-ever ischaemic and haemorrhagic stroke during 1990-2010: findings from the Global Burden of Disease Study 2010. Lancet Global Health 2013;1:e259-e281.

72. Auriat AM, Wowk S, Colbourne F. Rehabilitation after intracerebral hemorrhage in rats improves recovery with enhanced dendritic complexity but no effect on cell proliferation. Behav Brain Res 2010;214:42-47.

73. Santos M, Pagnussat A, Mestriner R, Netto C. Motor skill training promotes sensorimotor recovery and increases microtubuleassociated protein-2 (MAP-2) immunoreactivity in the motor cortex after intracerebral hemorrhage in the rat. ISRN Neurol 2013;2013:159184.

74. Liang H, Yin Y, Lin T, et al. Transplantation of bone marrow stromal cells enhances nerve regeneration of the corticospinal tract and improves recovery of neurological functions in a collagenase- 
induced rat model of intracerebral hemorrhage. Mol Cells 2013;36:17-24.

75. Hays SA, Khodaparast N, Hulsey DR, et al. Vagus nerve stimulation during rehabilitative training improves functional recovery after intracerebral hemorrhage. Stroke 2014;45:10-3097-3100.

76. Faul M, Coronado V. Epidemiology of traumatic brain injury. Handb Clin Neurol 2015;127:3-13.

77. Brown AW, Malec JF, Diehl NN, Englander J, Cifu DX. Impairment at rehabilitation admission and 1 year after moderate-to-severe traumatic brain injury: a prospective multicentre analysis. Brain Injury 2007;21:673-680.

78. Walker WC, Pickett TC. Motor impairment after severe traumatic brain injury: a longitudinal multicenter study. J Rehab Res Dev 2007;44:975-982.

79. Nishibe M, Barbay S, Guggenmos D, Nudo RJ. Reorganization of motor cortex after controlled cortical impact in rats and implications for functional recovery. J Neurotrauma 2010;27:2221-2232.

80. Axelson HW, Winkler T, Flygt J, Djupsjö A, Hånell A, Marklund N. Plasticity of the contralateral motor cortex following focal traumatic brain injury in the rat. Restorative Neurol Neurosci 2013;31: 73-85.

81. Jefferson SC, Clayton ER, Donlan NA, Kozlowski DA, Jones TA, Adkins DL. Cortical stimulation concurrent with skilled motor training improves forelimb function and enhances motor cortical reorganization following controlled cortical impact. Neurorehabil Neural Repair 2015 Aug 5 [Epub ahead of print].

82. Pruitt D, Schmid A, Kim L, et al. Vagus nerve stimulation delivered with motor training enhances recovery of function after traumatic brain injury. J Neurotrauma 2015 Aug 5 [Epub ahead of print].

83. Raineteau O, Schwab ME. Plasticity of motor systems after incomplete spinal cord injury. Nat Rev Neurosci 2001;2:263-273.

84. Navarro X, Vivó M, Valero-Cabré A. Neural plasticity after peripheral nerve injury and regeneration. Prog Neurobiol 2007;82: 163-201.

85. Udina E, Cobianchi S, Allodi I, Navarro X. Effects of activitydependent strategies on regeneration and plasticity after peripheral nerve injuries. Ann Anat 2011;193:347-353.

86. Brown AR, Hu B, Antle MC, Teskey GC. Neocortical movement representations are reduced and reorganized following bilateral intrastriatal 6-hydroxydopamine infusion and dopamine type-2 receptor antagonism. Exp Neurol 2009;220:162-170.

87. Brown AR, Antle MC, Hu B, Teskey GC. High frequency stimulation of the subthalamic nucleus acutely rescues motor deficits and neocortical movement representations following 6hydroxydopamine administration in rats. Exp Neurol 2011;231: 82-90.

88. Viaro R, Morari M, Franchi G. Progressive motor cortex functional reorganization following 6-hydroxydopamine lesioning in rats. J Neurosci 2011;31:4544-4554.

89. Plowman EK, Maling N, Thomas NJ, Fowler SC, Kleim JA. Targeted motor rehabilitation dissociates corticobulbar versus corticospinal dysfunction in an animal model of Parkinson's disease. Neurorehabil Neural Repair 2014;28:85-95.

90. Braak H, Del Tredici K, Rüb U, de Vos RA, Steur ENJ, Braak E. Staging of brain pathology related to sporadic Parkinson's disease. Neurobiol Aging 2003;24:197-211.

91. Del Tredici K, Braak H. Dysfunction of the locus coeruleusnorepinephrine system and related circuitry in Parkinson's disease-related dementia. J Neurol Neurosurg Psychiatry 2013;84:774-783.

92. Hoge CW, Castro CA, Messer SC, McGurk D, Cotting DI, Koffman RL. Combat duty in Iraq and Afghanistan, mental health problems, and barriers to care. N Engl J Med 2004;351:13-22.
93. Bremner JD, Elzinga B, Schmahl C, Vermetten E. Structural and functional plasticity of the human brain in posttraumatic stress disorder. Prog Brain Res 2007;167:171-186.

94. Peña DF, Engineer ND, McIntyre CK. Rapid remission of conditioned fear expression with extinction training paired with vagus nerve stimulation. Biol Psychiatry 2012;73:1071-1077.

95. Sandkühler J, Lee J. How to erase memory traces of pain and fear. Trends Neurosci 2013;36:343-352.

96. Foa E, Hembree E, Rothbaum BO. Prolonged exposure therapy for PTSD: emotional processing of traumatic experiences therapist guide. Oxford University Press, Oxford, 2007.

97. Powers MB, Halpern JM, Ferenschak MP, Gillihan SJ, Foa EB. A meta-analytic review of prolonged exposure for posttraumatic stress disorder. Clin Psychol Rev 2010;30:635-641.

98. Fanselow MS. Fear and anxiety take a double hit from vagal nerve stimulation. Biol Psychiatry 2013;73:1043-1044.

99. Furmaga H, Shah A, Frazer A. Serotonergic and noradrenergic pathways are required for the anxiolytic-like and antidepressantlike behavioral effects of repeated vagal nerve stimulation in rats. Biol Psychiatry 2011;70:937-945.

100. George MS, Ward HE, Jr, Ninan PT, et al. A pilot study of vagus nerve stimulation (VNS) for treatment-resistant anxiety disorders. Brain Stimul 2008;1:112-121.

101. Peña DF, Childs JE, Willett S, Vital A, McIntyre CK, Kroener S. Vagus nerve stimulation enhances extinction of conditioned fear and modulates plasticity in the pathway from the ventromedial prefrontal cortex to the amygdala. Front Behav Neurosci 2014;8: 327.

102. Marek R, Strobel C, Bredy TW, Sah P. The amygdala and medial prefrontal cortex: partners in the fear circuit. J Physiol (Lond) 2013;591:2381-2391.

103. Milad MR, Quirk GJ. Neurons in medial prefrontal cortex signal memory for fear extinction. Nature 2002;420:70-74.

104. Maren S, Quirk GJ. Neuronal signalling of fear memory. Nat Rev Neurosci 2004;5:844-852.

105. Brunoni AR, Lopes M, Fregni F. A systematic review and metaanalysis of clinical studies on major depression and BDNF levels: implications for the role of neuroplasticity in depression. Int $\mathrm{J}$ Neuropsychopharmacol 2008;11:1169.

106. Huber KM, Gallagher SM, Warren ST, Bear MF. Altered synaptic plasticity in a mouse model of fragile $\mathrm{X}$ mental retardation. Proc Natl Acad Sci U S A 2002;99:7746-7750.

107. Moretti P, Levenson JM, Battaglia F, et al. Learning and memory and synaptic plasticity are impaired in a mouse model of Rett syndrome. J Neurosci 2006;26:319-327.

108. Waung MW, Huber KM. Protein translation in synaptic plasticity: mGluR-LTD, Fragile X. Curr Opin Neurobiol 2009;19:319-326.

109. Bird LM. Angelman syndrome: review of clinical and molecular aspects. Appl Clin Genet 2014;7:93.

110. Hanks SB. Motor disabilities in the Rett syndrome and physical therapy strategies. Brain Develop 1990;12:157-161.

111. Dawson G, Rogers S, Munson J, et al. Randomized, controlled trial of an intervention for toddlers with autism: the Early Start Denver Model. Pediatrics 2010;125:e17-23.

112. Warren Z, McPheeters ML, Sathe N, Foss-Feig JH, Glasser A, Veenstra-Vanderweele J. A systematic review of early intensive intervention for autism spectrum disorders. Pediatrics 2011;127: e1303-e1311.

113. Sawaki L, Yaseen Z, Kopylev L, Cohen LG. Age-dependent changes in the ability to encode a novel elementary motor memory. Ann Neurol 2003;53:521-524.

114. Müller-Dahlhaus JFM, Orekhov Y, Liu Y, Ziemann U. Interindividual variability and age-dependency of motor cortical plasticity induced by paired associative stimulation. Exp Brain Res 2008;187:467-475. 
115. Pascual-Leone A, Freitas C, Oberman L, et al. Characterizing brain cortical plasticity and network dynamics across the agespan in health and disease with TMS-EEG and TMS-fMRI. Brain Topogr 2011;24:302-315.

116. Freitas C, Perez J, Knobel M, et al. Changes in cortical plasticity across the lifespan. Front Aging Neurosci 2011;3:5.

117. O'Donnell MJ, Xavier D, Liu L, et al. Risk factors for ischaemic and intracerebral haemorrhagic stroke in 22 countries (the INTERSTROKE study): a case-control study. Lancet 2010;376: 112-123.

118. Tomlinson B, Irving D, Blessed G. Cell loss in the locus coeruleus in senile dementia of Alzheimer type. J Neurol Sci 1981;49:419-428.

119. Yates C, Simpson J, Gordon A, et al. Catecholamines and cholinergic enzymes in pre-senile and senile Alzheimer-type dementia and Down's syndrome. Brain Res 1983;280:119-126.

120. German DC, Manaye KF, White CL, et al. Disease-specific patterns of locus coeruleus cell loss. Ann Neurol 1992;32:667-676.

121. Zweig R, Cardillo J, Cohen M, Giere S, Hedreen J. The locus ceruleus and dementia in Parkinson's disease. Neurology 1993;43:986-986.

122. Arango V, Underwood MD, John Mann J. Fewer pigmented neurons in the locus coeruleus of uncomplicated alcoholics. Brain Res 1994;650:1-8

123. Cullen K, Halliday G. Mechanisms of cell death in cholinergic basal forebrain neurons in chronic alcoholics. Metab Brain Dis 1995;10:81-91.

124. Cohen H, Benjamin J, Geva AB, Matar MA, Kaplan Z, Kotler M. Autonomic dysregulation in panic disorder and in post-traumatic stress disorder: application of power spectrum analysis of heart rate variability at rest and in response to recollection of trauma or panic attacks. Psychiatry Res 2000;96:1-13.

125. Zarow C, Lyness SA, Mortimer JA, Chui HC. Neuronal loss is greater in the locus coeruleus than nucleus basalis and substantia nigra in Alzheimer and Parkinson diseases. Arch Neurol 2003;60: 337.

126. Bracha HS, Garcia-Rill E, Mrak RE, Skinner R. Postmortem locus coeruleus neuron count in three American veterans with probable or possible war-related PTSD. J Neuropsychiatry Clin Neurosci 2005;17:503-509.

127. Krahl SE, Senanayake SS, Handforth A. Destruction of peripheral C-fibers does not alter subsequent vagus nerve stimulation-induced seizure suppression in rats. Epilepsia 2001;42:586-589.

128. Ruffoli R, Giorgi FS, Pizzanelli C, Murri L, Paparelli A, Fornai F. The chemical neuroanatomy of vagus nerve stimulation. J Chem Neuroanat 2011;42:288-296

129. Evans M, Verma-Ahuja S, Naritoku D, Espinosa J. Intraoperative human vagus nerve compound action potentials. Acta Neurol Scand 2004;110:232-238.

130. Verlinden T, Rijkers K, Hoogland G, Herrler A. Morphology of the human cervical vagus nerve: implications for vagus nerve stimulation treatment. Acta Neurol Scand 2015 Jul 20 [Epub ahead of print].

131. Castoro MA, Yoo PB, Hincapie JG, et al. Excitation properties of the right cervical vagus nerve in adult dogs. Exp Neurol 2011;227: 62-68.

132. Mollet L, Raedt R, Delbeke J, et al. Electrophysiological responses from vagus nerve stimulation in rats. Int $\mathrm{J}$ Neural Syst 2013;23:1350027.

133. Borland MS, Vrana WA, Moreno NA, et al. Cortical map plasticity as a function of vagus nerve stimulation intensity. Brain Stimul 2015 Sep 9 [Epub ahead of print].
134. Robert MY, John D. The relation of strength of stimulus to rapidity of habit-formation. J Comp Neurol Psychol 1908;18:459-482.

135. Gold PE, van Buskirk R. Posttraining brain norepinephrine concentrations: correlation with retention performance of avoidance training and with peripheral epinephrine modulation of memory processing. Behav Biol 1978;23:509-520.

136. Oitzl M, Hasenöhrl R, Huston J. Reinforcing effects of peripherally administered substance $P$ and its $C$-terminal sequence pGlu6SP6-11 in the rat. Psychopharmacology (Berl) 1990;100:308-315.

137. Baldi E, Bucherelli C. The inverted "u-shaped" dose-effect relationships in learning and memory: modulation of arousal and consolidation. Nonlinearity Biol Toxicol Med 2005;3:9-21.

138. McIntyre CK, McGaugh JL, Williams CL. Interacting brain systems modulate memory consolidation. Neurosci Biobehav Rev 2012;36:1750-1762

139. Van der Zee E, Luiten P. Muscarinic acetylcholine receptors in the hippocampus, neocortex and amygdala: a review of immunocytochemical localization in relation to learning and memory. Prog Neurobiol 1999;58:409-471.

140. Gainetdinov RR, Premont RT, Bohn LM, Lefkowitz RJ, Caron MG. Desensitization of G protein-coupled receptors and neuronal functions. Annu Rev Neurosci 2004;27:107-144.

141. Ernfors P, Bramham CR. The coupling of a trkB tyrosine residue to LTP. Trends Neurosci 2003;26:171-173.

142. Mattson MP, Maudsley S, Martin B. BDNF and 5-HT: a dynamic duo in age-related neuronal plasticity and neurodegenerative disorders. Trends Neurosci 2004;27:589-594.

143. Bramham CR, Messaoudi E. BDNF function in adult synaptic plasticity: the synaptic consolidation hypothesis. Prog Neurobiol 2005;76:99-125.

144. Bramham CR, Worley PF, Moore MJ, Guzowski JF. The immediate early gene arc/arg3. 1: regulation, mechanisms, and function. J Neurosci 2008;28:11760-11767.

145. Gottschalk WA, Jiang H, Tartaglia N, Feng L, Figurov A, Lu B. Signaling mechanisms mediating BDNF modulation of synaptic plasticity in the hippocampus. Learn Mem 1999;6:243-256.

146. Thomas GM, Huganir RL. MAPK cascade signalling and synaptic plasticity. Nat Rev Neurosci 2004;5:173-183.

147. Furmaga H, Carreno FR, Frazer A. Vagal nerve stimulation rapidly activates brain-derived neurotrophic factor receptor TrkB in rat brain. PLoS One 2012;7:e34844.

148. Dan Y, Poo M. Spike timing-dependent plasticity of neural circuits. Neuron 2004;44:23-30.

149. Seol GH, Ziburkus J, Huang SY, et al. Neuromodulators control the polarity of spike-timing-dependent synaptic plasticity. Neuron 2007;55:919-929.

150. Zhang J, Zhang J, The influence of vagus nerve stimulation on NMDAR1 mRNA and GABAAR alpha 1 mRNA in thalamic reticular neucus of pentylenetetrazole-induced epileptic rats. Sheng Wu Yi Xue Gong Cheng Xue Za Zhi 2002;19: 566-568.

151. Zagon A, Kemeny AA. Slow hyperpolarization in cortical neurons: a possible mechanism behind vagus nerve simulation therapy for refractory epilepsy? Epilepsia 2000;41:13821389.

152. Valdés-Cruz A, Magdaleno-Madrigal VM, Martínez-Vargas D, Fernández-Mas R, Almazán-Alvarado S. Long-term changes in sleep and electroencephalographic activity by chronic vagus nerve stimulation in cats. Prog Neuropsychopharmacol Biol Psychiatry 2008;32:828-834 